\title{
Etude M.E.H.R. de la martensite $\varepsilon$ thermique dans un alliage Fe-Mn-Si-Cr-Ni à mémoire de forme
}

\author{
J.L. PUTAUX ${ }^{*}(1)$, L. FEDERZONI ${ }^{* * *}$, M. MANTEL ${ }^{* *}$, G. BLANC ${ }^{* *}$ et J.P. CHEVALIER ${ }^{*}$ \\ * Centre d'Etudes de Chimie Métallurgique, CNRS, 15 Rue Georges Urbain, 94407 Vitry cedex, France \\ ** Centre de Recherche d'Ugine, Société Ugine Savoie, 73403 Ugine cedex, France \\ ${ }^{* * *}$ GEMPPM, Bât. 502, INSA, 69621 Villeurbanne cedex, France
}

\begin{abstract}
RESUME: Des échantillons polycristallins d'un alliage à mémoire de forme Fe-Mn-Si-Cr-Ni, contenant $70 \%$ de martensite $\varepsilon$ thermique, ont été étudiés par M.E.H.R. Les images montrent, à l'échelle atomique, la structure feuilletée de la phase $\varepsilon$. A partir d'images traitées de l'extrémité de plaquettes $\varepsilon$ élémentaires, nous avons analysé la structure de l'interface $\varepsilon$ (h.c.) $/ \gamma$ (c.f.c.). Celle-ci peut être décrite au moyen d'une distribution périodique de partielles de Shockley à $30^{\circ}$ et $90^{\circ}$ dans le rapport $2 / 1$. Des calculs de relaxation élastique ont confirmé ce modèle qui traduit les propriétés d'auto-accommodation de la martensite $\varepsilon$ thermique à l'échelle la plus fine.
\end{abstract}

\begin{abstract}
Polycrystalline samples of the Fe-Mn-Si-Cr-Ni shape memory alloy which contained a $70 \%$ fraction of thermal $\varepsilon$-martensite, have been studied by H.R.E.M. Images taken along a $<011>\gamma / /<2110>\varepsilon$ axis clearly show, at the atomic level, the layered substructure of the $\varepsilon$ phase. The structure of the $\varepsilon$ (h.c.p.) $/ \gamma$ (f.c.c.) interface has been analysed from numerically processed images of elementary $\varepsilon$-laminate ends. It can be described by a periodic array of $30^{\circ}$ and $90^{\circ}$ Shockley partials in a 2:1 ratio. Isotropic elasticity calculations confirmed this model which illustrates the self-accommodation properties of thermal $\varepsilon$-martensite at the finest scale.
\end{abstract}

\section{INTRODUCTION}

L'alliage $\mathrm{Fe}-\mathrm{Mn}-\mathrm{Si}-\mathrm{Cr}-\mathrm{Ni}$ subit une transformation martensitique de l'austénite $\gamma$ (cubique à faces centrées) en martensite $\varepsilon$ (hexagonale compacte) $[\mathbf{1 , 2}]$. On distingue la martensite thermique, formée par refroidissement de l'alliage en dessous de la température $\mathrm{M}_{\mathrm{S}}$, et la martensite de déformation, obtenue par déformation du matériau au-dessus de la température $\mathrm{M}_{\mathrm{S}}$. La transformation martensitique c.f.c. $\rightarrow$ h.c., qui s'effectue par glissement de partielles de Shockley $a / 6<112>$ tous les deux plans $\{111\} \gamma$, a été étudiée en détail par microscopie électronique en transmission dans différents aciers [3 à 8] ainsi que dans Co [9]. Le mouvement réversible des Shockley jouerait un rôle important dans les phénomènes de mémoire de forme de ces alliages. Dans cet article, nous présenterons plusieurs résultats obtenus par M.E.H.R. concernant la transformation martensitique $\gamma$ (c.f.c.) $\rightarrow \varepsilon$ (h.c.) dans des échantillons polycristallins d'un alliage élaboré par Ugine Savoie, de composition (en poids\%): Fe - 15,9 Mn - 5 Si - 9,1 Cr - 4,2 Ni.

\section{METHODE EXPERIMENTALE}

Federzoni et al [10] ont récemment mis ont point un traitement thermomécanique qui permet d'obtenir un rendement optimum en martensite thermique à partir de l'alliage brut de coulée. Ce traitement est le suivant:

- étape 1: laminage à froid + recuit $850^{\circ} \mathrm{C}(30 \mathrm{mn}$, air $)+$ refroidissement lent

- étape 2: déformation $7 \%$ + recuit $800^{\circ} \mathrm{C}(5 \mathrm{mn}$, air $)+$ refroidissement $\mathrm{N}_{2}$ liquide

(1) Adresse actuelle : CERMAV, BP. 53X, 38041 Grenoble cedex, France 

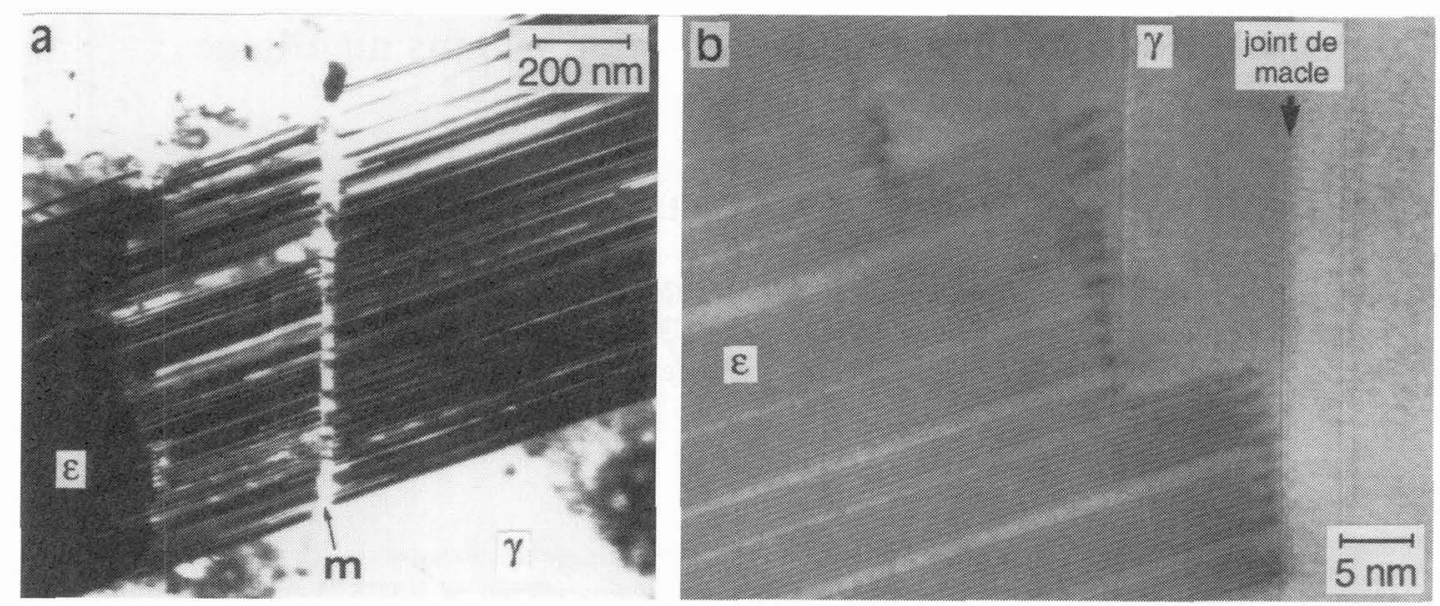

Fig.I. a) Structure feuilletée d'une large plaquette $\varepsilon$ thermique traversée par une micromacle $m$ de phase $\gamma$ (fond noir à partir d'une tache (111) commune à la macle et à la matrice $\gamma$ apparaissant en clair).

b) micrographie haute résolution de la zone d'interaction entre la phase $\varepsilon$ et la micromacle de phase $\gamma$.

Après le premier recuit de recristallisation, le matériau refroidi contient $4 \%$ de phase $\varepsilon$ thermique. Après l'étape 2, il en contient 70\%. Les éprouvettes sont alors amincies, découpées en pastilles de $3 \mathrm{~mm}$ de diamètre et $150 \mu \mathrm{m}$ d'épaisseur puis percées électrolytiquement. Les lames minces sont ensuite observées dans un microscope TOPCON ABT 002B $(200 \mathrm{kV})$ à ultra haute résolution.

\section{RESULTATS}

\subsection{Microscopie électronique}

La phase $\varepsilon$ se présente sous la forme de plaquettes parallèles aux plans $\{111\}$ de l'austénite $\gamma$. Ces plaquettes sont imparfaites. En particulier, lorsqu'elles sont orientées selon une direction proche de $<011>\gamma / /<2110>\varepsilon$ (fig. 1a), on observe de nombreux défauts parallèles aux plans de base et introduisant des traînées diffuses dans le diagramme de diffraction. Comme l'avaient déjà observé Yang et al [11], la martensite $\varepsilon$ thermique possède une structure feuilletée. Une plaquette épaisse est en fait constituée de nombreuses plaquettes plus fines séparées par de l'austénite résiduelle.

La figure $1 \mathrm{~b}$ montre, à haute résolution, la zone d'interaction entre une plaquette $\varepsilon$ et une micromacle $\gamma$, orientées selon un axe $\left\langle 011>_{\gamma} / /\langle 2110\rangle_{\varepsilon}\right.$. Le feuilletage de la martensite apparaît à l'échelle atomique. On voit un ensemble de fines plaquettes $\varepsilon$, de quelques dizaines de plans atomiques d'épaisseur, incluses dans de l'austénite résiduelle. On distingue l'empilement A-B-C des plans $\{111\}$ de la phase c.f.c. $\left(\mathrm{d}_{111}=0,207\right.$ $\mathrm{nm}$ avec a $=0,359 \mathrm{~nm}[12])$. Par contre, pour la phase hexagonale qui apparaît plus sombre, seule une famille de plans $\{0002\}$ de l'empilement $A-B$ est clairement imagée ( $d_{0002}=0,205 \mathrm{~nm}$ avec $a_{\varepsilon}=0,253 \mathrm{~nm}$ et $\left.c_{\varepsilon}=0,411 \mathrm{~nm}[12]\right)$. Cette surintensité est souvent observée sur des images de structures hexagonales [13].

L'interface $\varepsilon / \gamma$ à l'extrémité d'une fine plaquette, généralement perpendiculaire au grand axe de celle-ci (fig. 1b), présente des contrastes sombres qui lui donnent un aspect de peigne. Il a été nécessaire de traiterles micrographies très bruitées par filtrage numérique, en ne conservant pour construire l'image que l'intensité des faisceaux diffractés (à l'exception de taches (0001)ع "interdites", ceci pour supprimer l'effet de surintensité un plan sur deux) et des traînées diffuses (fig. 2b). Par ailleurs, au moyen de simulations d'images haute résolution, nous avons déterminé que les atomes apparaissaient vraisemblablement en blanc. Le filtrage révêle que les contrastes sombres correspondent à de petites fautes d'empilement intrinsèques qui s'étendent hors de l'interface sur quelques distances interatomiques (fig. $2 \mathrm{a}$ et $2 \mathrm{c}$ ). En général, ces fautes 

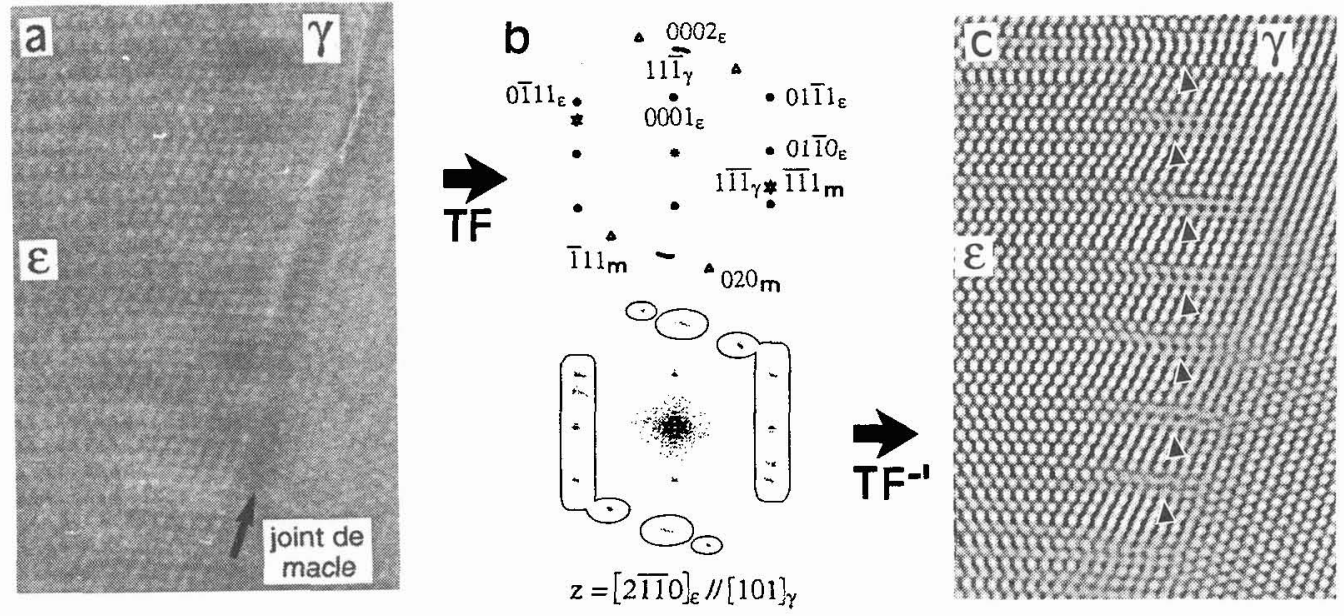

Fig.2. a) Image M.E.H.R. de l'interface $\varepsilon / \gamma$ en bout de plaquette.

b) Spectre de puissance de l'image a: en haut, les taches sont indentifiées par rapport aux familles de plans diffractants correspondantes. En bas sont représentés les masques utilisés pour le filtrage.

c) Image a traitée. Les flèches désignent les petites fautes d'empilement émergeant de l'interface.

sont réparties tous les 6 plans $\{111\} \gamma / /\{0002\}_{\varepsilon}$. Certaines déformations de plans atomiques doivent aussi être soulignées. D'une part, les plans $(0002)_{\varepsilon}$, cohérents avec les plans $(111) \gamma$, sont légèrement distordus au passage de l'interface. D'autre part, près de l'interface, les plans (111) $\gamma$ s'orientent parallèlement aux plans pyramidaux $(0111)_{\varepsilon}$.

Dans le cas d'observations selon la direction [101], seules les dislocations dont la ligne est parallèle à cet axe peuvent être étudiées a haute résolution. Trois d'entre elles sont alors susceptibles de transformer l'austénite c.f.c. en cristal h.c. (fig. 3): deux partielles de Shockley à $30^{\circ}$ (de composantes vis opposées) et une à $90^{\circ}$ (de composante coin opposée à celle des $30^{\circ}$ ). La somme des trois vecteurs de Burgers est nulle. Selon l'axe [101], on ne détecte que les composantes coin, ce qui donne l'impression de ne disposer que de deux types de Shockley de vecteur de Burgers: $\mathbf{b}_{90}$ et $-\mathbf{b}_{30}{ }^{ \pm}$.

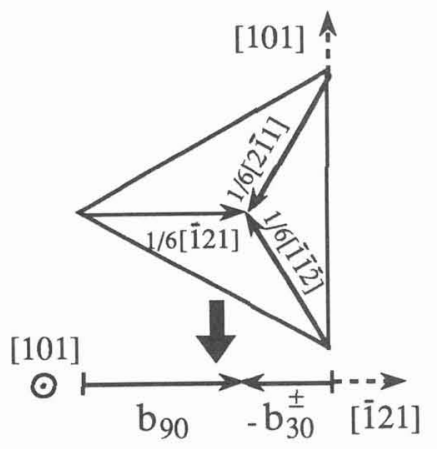

Fig.3. Partielles de Shockley assurant la transformation $\gamma \rightarrow \varepsilon$ et observables à haute résolution. Les vecteurs de Burgers sont représentés dans le plan (111) puis projetés selon la direction [101].
A l'aide d'un circuit de Burgers classique, nous avons identifié les dislocations bordant les fautes d'empilement: ce sont des Shockley à $90^{\circ}$ mais nous n'avons pu faire de même pour caractériser les autres dislocations. Nous avons donc utilisé la méthode de Howe et al [14], proposée pour des interfaces c.f.c. / h.c. dans des alliages Al-Ag. Les auteurs ont défini deux circuits permettant de discriminer les Shockley à $30^{\circ}$ et $90^{\circ}$. Cette méthode présuppose que l'interface contienne uniquement ce type de dislocation.

La figure 4 montre le résultat de la méthode appliquée à une image filtrée. Les circuits englobent une portion d'interface de 30 plans atomiques avec une périodicité d'une faute d'empilement tous les 6 plans. Le circuit 1 permet de trouver un défaut de fermeture $S F^{\prime}$ équivalent à 10 dislocations $-b_{30} \pm$. Le circuit 2 donne un défaut de fermeture SF" correspondant à 5 dislocations $b_{90}$. On dénombre bien 15 dislocations pour 30 plans et l'on obtient la proportion: $n\left(-b_{30}^{ \pm}\right) / n\left(b_{90}\right)=2 / 1$. 
Même si nous n'avons pas accès au sens de la composante vis des partielles à $30^{\circ}$, il est peu probable que l'interface ne contienne que des composantes vis de même sens. Cela introduirait une composante de torsion et des déformations importantes qui affecteraient nettement l'image. Les deux types de partielles à $30^{\circ}$ sont vraisemblablement en nombre égal, ceci pour annuler l'effet des composantes vis. La proportion des Shockley est finalement: $\mathbf{n}\left(-\mathbf{b}_{30^{-}}\right)=\mathbf{n}\left(\cdot \mathbf{b}_{30}{ }^{+}\right)=\mathbf{n}\left(\mathbf{b}_{90}\right)$. Le vecteur de Burgers global sur cette portion d'interface périodique est donc nul.

Fig.4. Détermination par la méthode de Howe et al [14] de la proportion de partielles de Shockley a $30^{\circ}$ et $90^{\circ}$ contenues dans l'interface $\varepsilon / \gamma$.

$$
\begin{gathered}
\overrightarrow{S F}^{\prime} \Rightarrow 10 \times\left(\vec{b}_{30}^{ \pm}\right) \\
\overrightarrow{\mathrm{SF}^{\prime \prime}} \Rightarrow 5 \times\left(\overrightarrow{\mathrm{b}}_{90}\right)
\end{gathered}
$$

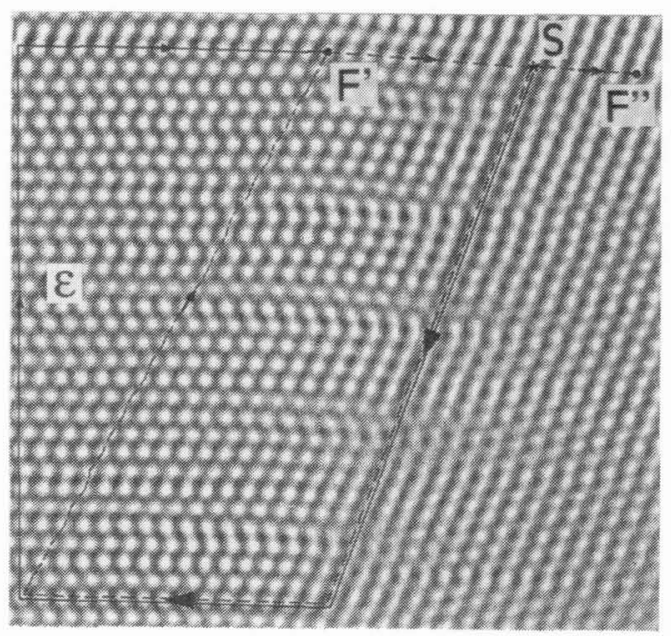

\subsection{Calculs de relaxation élastique}

Nous avons restreint la simulation au cas isotrope qui a le mérite d'être simple dans son formalisme mais reste approximatif et, en particulier, ne permet pas de retrouver une variation de paramètre cristallin liée au changement de phase. Le but étant de comparer les modèles obtenus à des images expérimentales qui sont des projections des structures, nous avons ramené la relaxation à un calcul bidimensionnel. La cellule initiale est un cristal c.f.c. projeté selon la direction [101]. Des dislocations de ligne [101] et de vecteurs de Burgers b sont introduites dans cette cellule, leur origine étant repérée par rapport à certains atomes. Les déplacements ux et uy autour des dislocations sont alors déterminés au moyen des équations de Hirth et Lothe [15]. Le calcul ne tient compte que des composantes coin des vecteurs de Burgers.

Nous avons simulé la transformation martensitique en plaçant dans la cellule non relaxée plusieurs dislocations à $30^{\circ}$ et $90^{\circ}$ tous les deux plans $\{111\} \gamma$ (fig. 5a). Le modèle obtenu (fig. 5b) pour une répartition de Shockley identique à celle déterminée à partir des images expérimentales filtrées reproduit de manière satisfaisante l'ensemble des déformations caractéristiques des plans $\{111\}$ soulignées dans le paragraphe 3.1. La superposition du modèle relaxé à une micrographie filtrée conduit à un bon accord. Bien que nous n'ayons pas calculé son énergie, on peut considérer que le modèle "en peigne" permet, en première approximation, de minimiser l'énergie élastique à l'extrémité des plaquettes de phase $\varepsilon$.

\section{DISCUSSION}

\subsection{Nucléation et propagation de la phase hexagonale}

La répartition des Shockley à l'interface $\varepsilon / \gamma$ n'étant pas aléatoire, la croissance des plaquettes ne s'est donc pas faite par multiplication d'un seul type de dislocation à partir de pôles tels que ceux invoqués par Seeger [8]. Il n'y a pas eu non plus de nucléation au hasard et de recouvrement progressif de fautes d'empilement jusqu'à une répartition tous les deux plans. Les processus proposés par Fujita et al [3] et Inagaki $[4,5]$ pour décrire la formation de la martensite de déformation, ne peuvent pas conduire à la distribution périodique observée à l'extrémité des plaquettes de phase $\varepsilon$ thermique. 


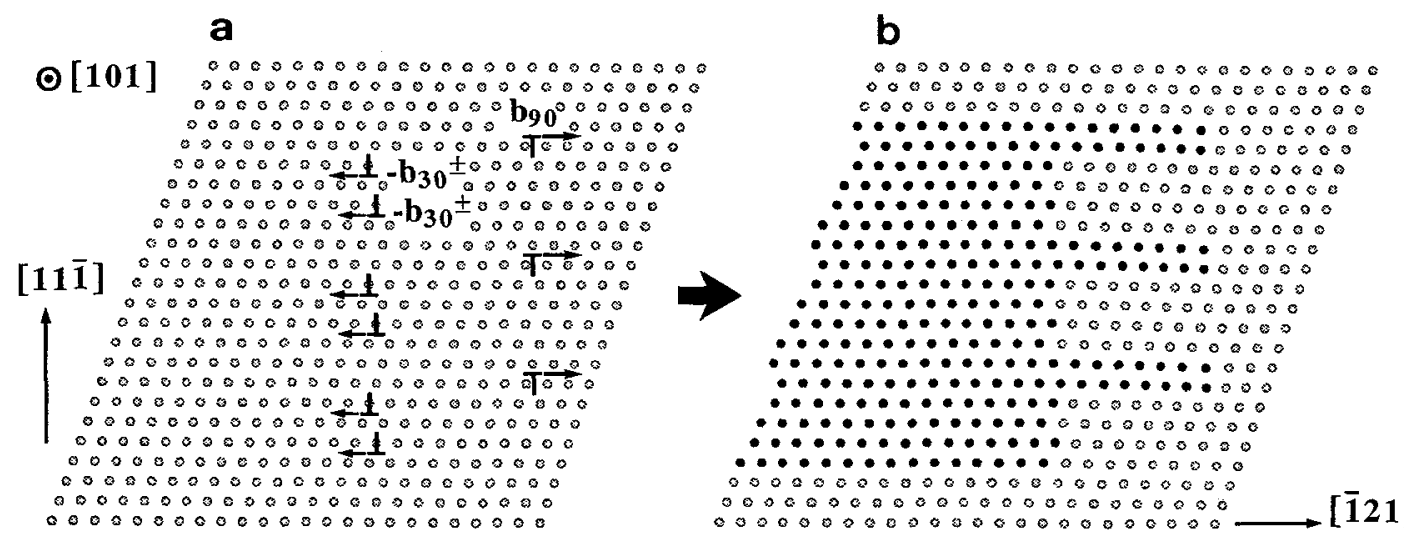

Fig.5. Calculs de relaxation élastique: a) cellule de calcul initiale non relaxée dans laquelle sont introduites 3 partielles à $90^{\circ}$ et 6 partielles a $30^{\circ}$ (les grandeurs des vecteurs de Burgers dessinés ne sont pas respectées).

b) cellule relaxée: les atomes qui suivent l'empilement hexagonal sont représentés en noir.

La répartition périodique des 3 types de partielles de Shockley ne peut être obtenue que si la nucléation et le mouvement des dislocations sont fortement corrélés. Ainsi, l'énergie élastique associée à la transformation est minimisée et la déformation globalement nulle, à l'échelle la plus petite. Puisque nous avons confirmé, à l'échelle atomique, la structure feuilletée de la phase $\varepsilon$ thermique, l'austénite se transforme vraisemblablement par propagation de fines plaquettes $\varepsilon$ bordées par un petit nombre de dislocations corrélées. La densification de la martensite se fait par nucléation de nouvelles plaquettes élémentaires plutôt que par épaississement de celles déjà existantes. La croissance latérale est difficile car la propagation des partielles parallèlement aux plans $\{111\} \gamma$ est rapide. La cinétique de la transformation est alors essentiellement gouvernée par la fréquence de germination des plaquettes et non par leur croissance.

\subsection{Epaisseur des plaquettes élémentaires et densité de la phase $\varepsilon$}

Il est clair que la contraction de la phase $\varepsilon$ normalement aux plans de base [12] a une influence sur l'épaisseur moyenne des plaquettes élémentaires et la croissance latérale des plaquettes. Plus l'épaisseur augmente, plus l'énergie accumulée à son extrémité devient élevée. Si la croissance latérale dépasse un certain seuil d'épaisseur, la différence de paramètre devra être accommodée par des dislocations de "misfit" dont le vecteur de Burgers est orienté selon la normale aux plans de base. Dans un cristal c.f.c., ces dislocations sont des partielles de Frank $\mathrm{a} / 3<111>$. Nous n'avons pas rencontré de telles dislocations.

Au cours de la phase de propagation, l'épaisseur des plaquettes doit rester inférieure à la valeur critique. En effet, une énergie élastique trop forte accumulée dans le front de la plaquette diminuerait la vitesse de propagation. De plus, les partielles de Frank, sessiles dans les plans $\{111\}_{\gamma}$ et $\{0002\} \varepsilon$, devraient se déplacer par montée, ce qui nécessiterait de la diffusion et irait donc à l'encontre d'un bon effet mémoire.

\subsection{Auto-accommodation de la martensite $\varepsilon$ thermique}

La figure 6 rappelle les deux manières de transformer un cristal c.f.c. en martensite h.c. La première (fig. 6a) se produit par propagation d'un seul type de dislocation, lorsque l'on déforme le cristal $\gamma$. La seconde (fig. $6 \mathrm{~b}$ ) correspond à l'alternance des trois cisaillements possibles dans une même famille de plans $\{111\} \%$. Ce processus, intervenant au cours d'une transformation spontanée, conduit à une déformation globalement nulle. Dans notre cas, un bloc de la figure $6 \mathrm{~b}$ correspond à deux plans atomiques cisaillés par une partielle de Shockley. L'auto-accommodation des déformations se fait donc à l'échelle de la dislocation. En étudiant un alliage de composition proche de celle de nos échantillons, Yang et al [11] ont constaté que 
Fig.6. Représentation schématique de la transformation d'un cristal c.f.c. en martensite h.c.:

a) croissance préférentielle d'un variant par déformation.

b) transformation spontanée auto-accommodée par l'alternance de 3 cisaillements. (d'après [11])

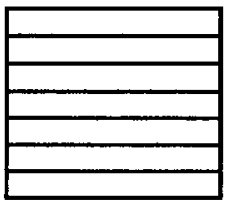

c.f.c.

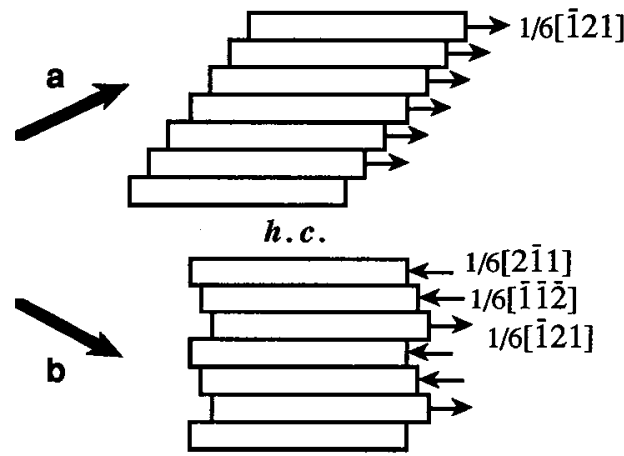

l'épaisseur des blocs pouvait fluctuer. Dans les larges bandes $\varepsilon$ qu'ils ont observées, la compensation des cisaillements est assurée par l'alternance de 3 variants d'épaisseur variable $(100$ à $150 \mathrm{~nm})$, chacun étant formé par la propagation d'un seul type de Shockley.

Nos résultats et ceux de Yang illustrent deux manières d'accommoder les déformations dans une plaquette $\varepsilon$. Dans un polycristal, l'auto-accommodation est sensible aux contraintes locales. La nature et la proportion des dislocations de transformation peuvent donc varier. L'auto-accommodation à l'échelle de la dislocation, dans nos échantillons, signifie que les contraintes résiduelles de l'alliage recristallisé sont minimisées.

\section{CONCLUSION}

Les observations à haute résolution de plaquettes de martensite $\varepsilon$ thermique ont confirmé, à l'échelle atomique, la structure feuilletée de cette phase. Elles ont de plus révélé que les interfaces entre les phases $\gamma$ et $\varepsilon$ étaient cohérentes et facettées parallèlement et perpendiculairement aux plans denses $(111) \gamma / /(0002)_{\varepsilon}$. La martensite se développe par nucléation et propagation de plaquettes élémentaires épaisses de quelques plans atomiques. Ces plaquettes se propagent par glissement corrélé de trois types de partielles de Shockley, en proportions égales et réparties périodiquement afin d'assurer la compensation des cisaillements. Ainsi, les déformations sont accommodées à l'échelle la plus fine. La densification de la phase $\varepsilon$ se fait par nucléation de nouvelles plaquettes élémentaires plutôt que par épaississement de celles déjà existantes.

\section{REFERENCES:}

[1] INAGAKI H., Z. Metallkd. 83-2 (1992) 90.

[2] YANG J.H., CHEN H., WAYMAN C.M., Metall. Trans. 23A (1992) 1431, 1439, 1445.

[3] FUJTA H., UEDA S., Acta Metall. 20 (1972) 759.

[4] INAGAKI H., Z. Metallkd. 83-2 (1992) 97.

[5] INAGAKI H., Z. Metallkd. 83-5 (1992) 304.

[6] HOSHINO Y., NAKAMURA S., ISHIKAWA N., YAMAJI Y., MATSUMOTO S., TANAKA Y., SATO A., Mat. Trans. JIM 33 (1992) 252.

[7] OLSON G.B., COHEN M., Metall. Trans. A. 7 (1976) 1897.

[8] SEEGER A., Z. Metallkd. 47 (1956) 653.

[9] MAHAJAN S., GREEN M.L., BRASEN D., Metall. Trans. 8A (1977) 283.

[10] FEDERZONI L., GEX D., GU Q., GUENIN G., LABROSSE D., MANTEL M., VAN HUMBEECK J., ICOMAT 92 , Monterey (USA).

[11] YANG J.H., WAYMAN C.M., Mater. Charact. 28 (1992) 23, 37.

[12] BLANC. G., communication privée.

[13] NAGAKURA S., NAKAMURA Y., SUZUKI T., Jpn. Journ. Appl. Phys. 21 (1982) 449.

[14] HOWE J.M., DAHMEN U., GRONSKY R., Phil. Mag. A 56 (1987) 31.

[15] HIRTH J.P., LOTHE J., Theory of Dislocations, McGraw-Hill 1968. 